\title{
GUT Baryogenesis after Preheating: Numerical Study of the Production and Decay of $X$-bosons
}

\author{
Edward W. Kolb ${ }^{a}$ 用, Antonio Riotto ${ }^{b}$ 畑 and Igor I. Tkachev ${ }^{c}$., \\ ${ }^{a}$ NASA/Fermilab Astrophysics Center \\ Fermilab National Accelerator Laboratory, Batavia, Illinois 60510-0500 \\ and \\ Department of Astronomy and Astrophysics \\ Enrico Fermi Institute, University of Chicago, Chicago, Illinois 60637-1433 \\ ${ }^{b}$ Theory Division, CERN, CH-1211 Geneva 23, Switzerland \\ ${ }^{c}$ Department of Physics, Purdue University, West Lafayette, Indiana 47907 \\ and \\ Institute for Nuclear Research of the Academy of Sciences of Russia \\ Moscow 117312, Russia
}

\begin{abstract}
We perform a fully non-linear calculation of the production of supermassive Grand Unified Theory (GUT) $X$ bosons during preheating, taking into account the fact that they are unstable with a decay width $\Gamma_{X}$. We show that parametric resonance does not develop if $\Gamma_{X}$ is larger than about $10^{-2} m_{X}$. We compute the nonthermal number density of superheavy bosons produced in the preheating phase and demonstrate that the observed baryon asymmetry may be explained by GUT baryogenesis after preheating if $\Gamma_{X}$ is smaller than about $10^{-3} m_{X}$.
\end{abstract}

PACS: $98.80 . \mathrm{Cq}$

\footnotetext{
${ }^{*}$ E-mail: rocky@rigoletto.fnal.gov

${ }^{\dagger}$ E-mail: riotto@nxth04.cern.ch

$\ddagger$ On leave from Department of Theoretical Physics, University of Oxford, U.K.

$\S$ E-mail: tkachev@physics . purdue.edu
} 


\section{Introduction}

The horizon and flatness problems of the standard big-bang cosmology are solved elegantly if during the evolution of the early universe the energy density happened to be dominated by some form of vacuum energy which resulted in a quasi-exponential growth of the scale factor [1]. An inflationary stage is also required to dilute any undesirable remnants such as topological defects surviving from some phase transition at a pre-inflation epoch.

The vacuum energy driving inflation is generally assumed to be associated with some scalar field $\phi$, known as the inflaton, which is initially displaced from the minimum of its potential. As a by-product of solving the horizon and flatness problem, quantum fluctuations of the inflaton field may produce the seeds necessary for the generation of structure formation and for fluctuations in the cosmic background radiation.

Inflation ended when the potential energy associated with the inflaton field became smaller than the kinetic energy of the field. By that time, any pre-inflation entropy in the universe had been inflated away, and the energy of the universe was entirely in the form of coherent oscillations of the inflaton condensate around the minimum of its potential. The universe may be said to be frozen after the end of inflation. We know that somehow the low-entropy cold universe dominated by the energy of coherent motion of the $\phi$ field must be transformed into a high-entropy hot universe dominated by radiation. The process by which the energy of the inflaton field is transferred from the inflaton field to radiation has been dubbed reheating. 1

In the old theory of reheating [2], it was assumed that the inflaton field oscillated around the minimum of its potential in a coherent way until the age of the universe grew to the order of the inflaton decay lifetime $\tau_{\phi}, t \sim \tau_{\phi}=\Gamma_{\phi}^{-1}$. At this stage, the inflaton decayed and the universe filled with the inflaton decay products, which soon thermalized. In the process the universe was "reheated" to the temperature of $T_{R H} \simeq 10^{-1} \sqrt{\Gamma_{\phi} M_{\mathrm{Pl}}}$, where $M_{\mathrm{Pl}} \sim 10^{19} \mathrm{GeV}$ is the Planck mass. In a simple chaotic inflation model the inflaton potential is given by $V(\phi)=m^{2} \phi^{2} / 2$, with $m \sim 10^{13} \mathrm{GeV}$ in

\footnotetext{
${ }^{1}$ Reheating may well be a misnomer since there is no guarantee that the universe was hot before inflation. Since we are confident that the universe was frozen at the end of inflation, perhaps "defrosting" is a better description of the process of converting inflaton coherent energy into entropy.
} 
order to reproduce the observed temperature anisotropies in the microwave background. Writing $\Gamma_{\phi}=\alpha_{\phi} m$, one finds $T_{R H} \simeq 10^{15} \sqrt{\alpha_{\phi}} \mathrm{GeV}$ [3].

The density and temperature fluctuations observed in the present universe, $\delta \rho / \rho \sim$ $10^{-5}$, require the inflaton potential to be extremely flat. This means that the couplings of the inflaton field to the other degrees of freedom (including $\alpha_{\phi}$ ) cannot be too large, since large couplings would induce large loop corrections to the inflaton potential, spoiling its flatness. As a result, $T_{R H}$ is expected to be smaller than $10^{14} \mathrm{GeV}$ by several orders of magnitude. The problem of large loop corrections to the inflaton potential may be solved in the framework of supersymmetry [4], where the nonrenormalization theorem [5] guarantees that the superpotential is not renormalized at any order of perturbation theory. On the other hand, in supergravity-inspired scenarios gravitinos have a mass of order a $\mathrm{TeV}$ and a decay lifetime on the order of $10^{5} \mathrm{~s}$. If gravitinos would be overproduced in reheating and decay after the epoch of nucleosynthesis, they would modify the successful predictions of big-bang nucleosynthesis. This can be avoided if the reheat temperature is smaller than about $10^{11} \mathrm{GeV}$ (or even less, depending on the gravitino mass).

In addition to entropy, the baryon asymmetry must be created after inflation. One method to generate the baryon asymmetry is by the decay of baryon-number $(B)$ violating superheavy bosons (referred to generically as " $X$ " bosons, whether gauge or Higgs bosons) of Grand Unified Theories (GUT's) [6]. In the old theory of reheating there is a serious obstacle to post-inflation GUT baryogenesis related to the relatively large $X$-boson mass and the relatively small reheat temperature.

The unification scale is generally assumed to be around $10^{16} \mathrm{GeV}$, and $B$-violating gauge bosons should have masses comparable to this scale. Baryon-number violating Higgs bosons may have a mass one or two orders of magnitude less. For example, in $S U(5)$ there are $B$ violating "Higgs" bosons in the five-dimensional representation that may have a mass as small as $10^{14} \mathrm{GeV}$. In fact, these Higgs bosons are more likely than gauge bosons to produce a baryon asymmetry since it is easier to arrange the requisite $\mathrm{CP}$ violation in the Higgs decay [7, 8, 9]. But even the light $B$-violating Higgs bosons are expected to have masses larger than the inflaton mass, and it would be kinematically impossible to create them directly in $\phi$ decay. 
One might think that the $X$ bosons could be created by thermal scattering during the stage of thermalization of the decay products of the inflaton field. However, if $T_{R H}$ is as small as necessary to avoid overproduction of gravitinos, production of superheavy bosons by thermal scattering would be heavily suppressed.[

But the outlook for GUT baryogenesis has brightened recently with the realization that reheating may differ significantly from the simple picture described above 111, 12, 13, 14, 15]. In the first stage of reheating, called "preheating" [11], nonlinear quantum effects may lead to an extremely effective dissipational dynamics and explosive particle production even when single particle decay is kinematically forbidden. Particles can be produced in the regime of a broad parametric resonance, and it is possible that a significant fraction of the energy stored in the form of coherent inflaton oscillations at the end of inflation is released after only a dozen or so oscillation periods of the inflaton. What is most relevant for the present discussion is that preheating may play an extremely important role for GUT generation of the baryon asymmetry. Indeed, it was shown in [16] that the baryon asymmetry can be produced efficiently just after the preheating era, thus solving many of the problems that GUT baryogenesis had to face in the old picture of reheating.

A crucial observation for baryogenesis is that even particles with mass larger than that of the inflaton may be produced during preheating. To see how this might work, let us assume that the interaction term between the superheavy bosons and the inflaton field is of the type $g^{2} \phi^{2}|X|^{2}$. During preheating, quantum fluctuations of the $X$ field with momentum $\vec{k}$ approximately obey the Mathieu equation: $X_{k}^{\prime \prime}+[A(k)-2 q \cos 2 z] X_{k}=0$, where $q=g^{2} \phi^{2} / 4 m^{2}, A(k)=\left(k^{2}+m_{X}^{2}\right) / m^{2}+2 q$, and primes denotes differentiation with respect to $z=m t$. Particle production occurs above the line $A=2 q$. The width of the instability strip scales as $q^{1 / 2}$ for large $q$, independent of the $X$ mass. The condition for broad resonance, $A-2 q \lesssim q^{1 / 2}[1]$, becomes $\left(k^{2}+m_{X}^{2}\right) / m^{2} \lesssim g \bar{\phi} / m$, which yields for the typical energy of $X$ bosons produced in preheating $E_{X}^{2}=k^{2}+m_{X}^{2} \lesssim g \bar{\phi} m$ [13], where $\bar{\phi}$ is the amplitude of the oscillating inflaton field. By the time the resonance

\footnotetext{
${ }^{2}$ There exists another problem for GUT baryogenesis scenarios: $B$ violation through sphaleron transitions are expected to be fast at high temperatures, and would erase any preexisting baryon asymmetry produced at the GUT scale [10] unless there is a non vanishing value of $B-L$. But a natural way to overcome this problem is to adopt a GUT like $S O(10)$, where an asymmetry in $B-L$ may be generated.
} 
develops to the full strength, $\bar{\phi}^{2} \sim 10^{-5} M_{\mathrm{Pl}}^{2}$. The resulting estimate for the typical energy of particles at the end of the broad resonance regime for $m \sim 10^{-6} M_{\mathrm{Pl}}$ is $E_{X} \sim$ $10^{-1} g^{1 / 2} \sqrt{m M_{\mathrm{Pl}}} \sim g^{1 / 2} 10^{15} \mathrm{GeV}$. Supermassive $X$ bosons can be produced by the broad parametric resonance for $E_{X}>m_{X}$, which leads to the estimate that $X$ production will be possible if $m_{X}<g^{1 / 2} 10^{15} \mathrm{GeV}$.

For $g^{2} \sim 1$ one would have copious production of $X$ particles (in this regime the problem is non-linear from the beginning and therefore $g^{2}=1$ has to be understood as a rough estimate of the limiting case) as heavy as $10^{15} \mathrm{GeV}$, i.e., 100 times greater than the inflaton mass. The only problem here is that for large coupling $g$, radiative corrections to the effective potential of the inflaton field may modify its shape at $\phi \sim M_{\mathrm{Pl}}$. However, this problem does not appear if the flatness of the inflaton potential is protected by supersymmetry.

This is a significant departure from the old constraints of reheating. Production of $X$ bosons in the old reheating picture was kinematically forbidden if $m<m_{X}$, while in the new scenario it is possible because of coherent effects. It is also important to note that the particles are produced out-of-equilibrium, thus satisfying one of the basic requirements to produce the baryon asymmetry [18].

Scattering of $\mathrm{X}$ fluctuations off the zero mode of the inflaton field limits the maximum magnitude of $\mathrm{X}$ fluctuations to be $\left\langle X^{2}\right\rangle_{\max } \approx m^{2} / g^{2}$ 14. For example, $\left\langle X^{2}\right\rangle_{\max } \sim$ $10^{-10} M_{\mathrm{Pl}}^{2}$ in the case $m_{X}=10 \mathrm{~m}$. This restricts the corresponding number density of created $X$-particles.

A potentially important dynamical effect is that the parametric resonance is efficient only if the self-interaction couplings of the superheavy particles are not too large. Indeed, a self-interaction term of the type $\lambda|X|^{4}$ provides a non-thermal mass to the $X$ boson of the order of $\left(\lambda\left\langle X^{2}\right\rangle\right)^{1 / 2}$, but this contribution is smaller than the bare mass $m_{X}$, if $\lambda \lesssim g^{2} m_{X}^{2} / m^{2}$. Self-interactions may also terminate the resonance effect because scattering induced by the coupling $\lambda$ may remove particles from the resonance shells and redistribute their momenta [16, 19. But this only happens if, again, $\lambda \gg g^{2}[13$.

The parametric resonance is also rendered less efficient when the $X$ particles have a large decay width $\Gamma_{X}$. Roughly speaking, one expects that the explosive production

\footnotetext{
${ }^{3}$ In the case in which the cross-coupling between the inflaton and the $X$ field is negative, superheavy particles may be produced even more efficiently [17].
} 
of particles takes place only if the typical time, $\tau_{e}$, during which the number of $X$ bosons grows by a factor of $e$, is smaller than the decay lifetime $\tau_{X}=\Gamma_{X}^{-1}$. During the broad resonance regime, typically $\tau_{e} \gtrsim 10 \mathrm{~m}^{-1}$. If we parameterize the decay width by $\Gamma_{X}=\alpha m_{X}$, this requires $\alpha \lesssim 0.1 m / m_{X}$. Notice that smaller values of $\Gamma_{X}$ are favored not only because particle production is made easier, but also because the superheavy particles may remain out-of-equilibrium for longer times, thus enhancing the final baryon asymmetry.

The exact knowledge of the maximum allowed value of the decay width of the superheavy degrees of freedom is therefore of extreme importance for the computation of the final baryon asymmetry produced by the GUT particles after preheating.

The goal of this paper is twofold. First, we wish to provide the first fully nonlinear calculation of the inflaton decay into superheavy $X$ bosons taking into account their decay width $\Gamma_{X}$. Our basic finding is that the parametric resonance does not develop if the decay rate $\Gamma_{X}$ is larger than about $10^{-1} \mathrm{~m}$, thus confirming the rough estimate made above. $X$ production through the resonance is very efficient for smaller values of $\Gamma_{X}$. Our second goal is to compute numerically the number density $n_{X}$ of supermassive $X$ bosons produced at the resonance stage. This parameter is fundamental for the computation of the final baryon asymmetry. We will also show that as long as the bound $\Gamma_{X} \lesssim 10^{-2} m$ is satisfied, the observed baryon asymmetry $B \simeq 4 \times 10^{-11}$ may be explained by the phenomenon of GUT baryogenesis after preheating, with no further restriction of the parameters. We will also comment on the phenomenological implications of our findings.

\section{$2 \quad X$ production and decay}

Using the methods developed in Refs. [12, 13, 14], we have studied numerically the production of massive, unstable $X$ particles in the process of the inflation decay.

We consider a model in which the oscillating inflaton field $\phi$ interacts with a scalar field $X$ whose decays violate baryon number $B$. A simple possibility for the $X$-particle is the Higgs field in the five-dimensional representation of $S U(5)$, although as noted above, because of the desirability of $B-L$ violation $S O(10)$ is a more promising theory.

\footnotetext{
${ }^{4}$ See also ref. [20].
} 
We assume standard kinetic terms, minimal coupling with gravity, and a very simple potential for the fields of the form

$$
V(\phi, X)=\frac{1}{2} m^{2} \phi^{2}+\frac{1}{2} m_{X}^{2} X^{2}+\frac{1}{2} g^{2} \phi^{2} X^{2} .
$$

Let us first introduce dimensionless variables in the conformal reference frame. The rescaled conformal time $\tau$ is related to cosmological time $t$ by $m d t=a(\tau) d \tau$. The rescaled conformal fields $\chi$ and $\varphi$ are related to the original fields by $X(\tau)=$ $\chi(\tau) \phi_{0}(0) / a(\tau)$ and $\phi(\tau)=\varphi(\tau) \phi_{0}(0) / a(\tau)$. In this model $\phi_{0}(0) \approx 0.28 M_{\mathrm{Pl}}$ is the value of the inflaton field at the end of inflation. We assume that immediately after inflation the universe is matter dominated and the scale factor evolves as $a(\tau)=$ $\left(\sqrt{\pi} \phi_{0}(0) \tau / \sqrt{3} M_{\mathrm{Pl}}+1\right)^{2} 13$.

An important dimensionless parameter in the problem will be the resonance parameter $q=g^{2} \phi_{0}^{2}(0) / 4 m^{2}$. For $m=1.3 \times 10^{-6} M_{\mathrm{Pl}}$ and $\phi_{0}(0)=0.28 M_{\mathrm{Pl}}, q \simeq 10^{10} g^{2}$.

In the conformal variables, the equations of motion become

$$
\begin{aligned}
\ddot{\varphi}-\nabla^{2} \varphi+\left(a^{2}-\frac{\ddot{a}}{a}\right) \varphi+4 q \chi^{2} \varphi & =0, \\
\ddot{\chi}-\nabla^{2} \chi+\Gamma a \dot{\chi}+\left(m_{\chi}^{2} a^{2}-\Gamma \dot{a}-\frac{\ddot{a}}{a}\right) \chi+4 q \varphi^{2} \chi & =0 .
\end{aligned}
$$

Here we are taking into account the decay of the $X$ field by simply introducing the term $\Gamma_{X} \dot{X}$ in the equation of motion for the field $X$. The dimensionless parameter $\Gamma$ which enters the Eq. (2) is $\Gamma \equiv \Gamma_{X} / m$. Similarly, $m_{\chi}=m_{X} / m$.

We have solved these equations of motion directly in coordinate space on a $128^{3}$ spatial lattice with periodic boundary conditions. The initial conditions for fluctuations correspond to the conformal vacuum at the time when the oscillations of $\varphi_{0}$ commence [12, 13]. The initial conditions for the coherently oscillating inflaton zero-momentum mode are $\varphi_{0}(0)=1, \dot{\varphi}_{0}(0)=0$.

A fundamental parameter in GUT baryogenesis is $n_{X}$, the number density of the supermassive leptoquarks whose decays produce the baryon asymmetry. It will depend upon the value of $\Gamma$ and $q$.

Since the supermassive bosons are more massive than the inflaton, one expects small kinetic energy in the excitations of the $X$ field. From the potential of Eq. (1), the square of the effective mass of the $X$ field is $\left(m_{X}^{\mathrm{EFF}}\right)^{2}=\left(m_{X}^{2}+g^{2}\left\langle\phi^{2}\right\rangle\right)$ and the energy density 


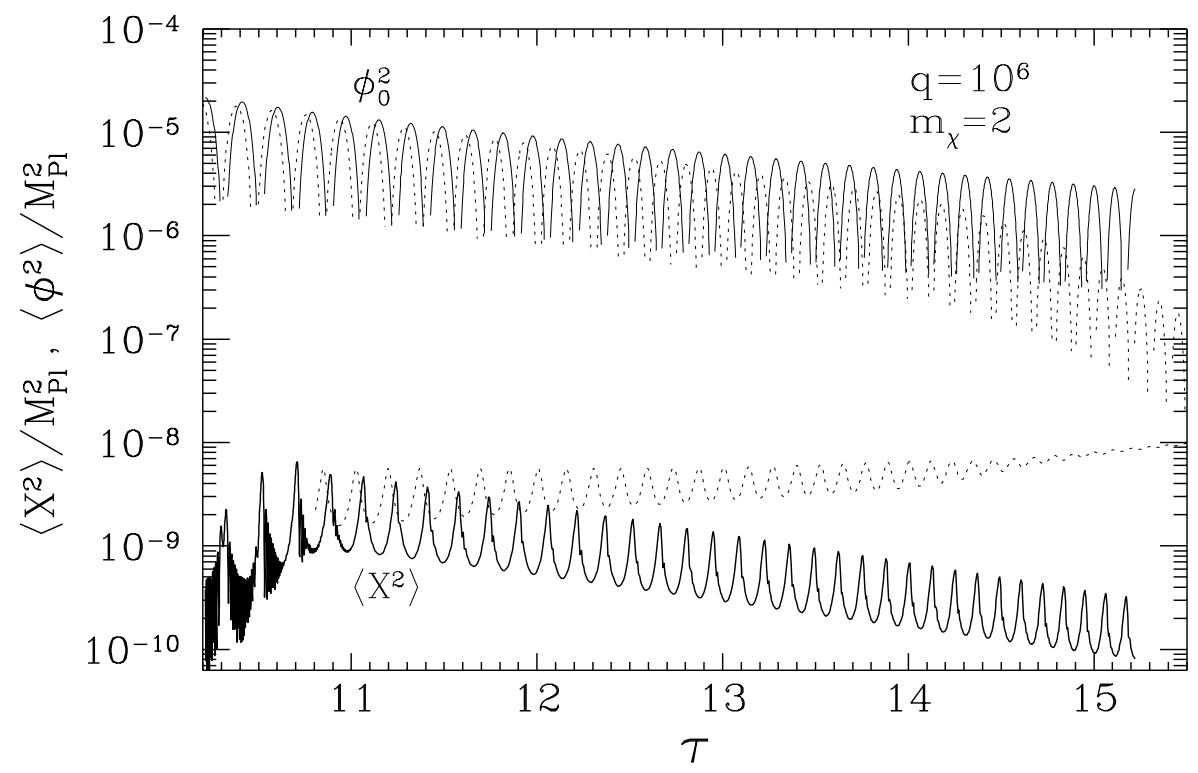

Figure 1: The variance of $X$ with model parameters $q=10^{6}, m_{\chi}=2$, and $\Gamma=6 \times 10^{-2}$ is shown by the lower solid curve as a function of time. The upper solid curve corresponds to the inflaton zero mode. The dotted curves represent the same quantities for $\Gamma=0$.

in the $X$ field will be $\rho_{X}=\left(m_{X}^{2}+g^{2}\left\langle\phi^{2}\right\rangle\right)\left\langle X^{2}\right\rangle$. Writing $\left\langle\phi^{2}\right\rangle$ as $\phi_{0}^{2}+\left\langle\delta \phi^{2}\right\rangle$, we can define an analog of the $X$-particle number density as

$$
n_{X}=\rho_{X} / m_{X}^{\mathrm{EFF}}=\left[4 q\left(\phi_{0}^{2}+\left\langle\delta \phi^{2}\right\rangle\right) / \phi_{0}^{2}(0)+m_{\chi}^{2}\right]^{1 / 2} m\left\langle X^{2}\right\rangle .
$$

Eq. ([3) enables us to calculate the number density of the created $X$-particles if the variances of the fields, $\left\langle X^{2}\right\rangle,\left\langle\delta \phi^{2}\right\rangle \equiv\left\langle[\phi(x)-\langle\phi\rangle]^{2}\right\rangle$, and the inflaton zero mode $\phi_{0}(\tau) \equiv$ $\langle\phi(\tau)\rangle$, are known. Here $\langle\cdots\rangle$ has to be understood as the average over statistical realizations. Since the system is homogeneous on average, this is equivalent to the volume average. We shall present the dependence upon the time of the variances for several choices of the model parameters as well as the maximum value of the variances which may be achieved during the evolution as a function of the same parameters.

The time evolution of the variance, $\left\langle X^{2}\right\rangle$, and of the inflaton zero mode, $\langle\phi\rangle$, is shown in Fig. 1 by the solid curves for the case $q=10^{6}, m_{\chi}=2$, and $\Gamma=6 \times 10^{-2}$. We see that the particle creation reaches a maximum at $\tau \approx 10.8$ when $\left\langle X^{2}\right\rangle \approx 10^{-9}$ in the "valleys" between the peaks. . At later times, $\tau>10.8$, particle creation by

\footnotetext{
${ }^{5}$ Note that we can use Eq. (3) only for the "valley" values of the variance, where the adiabatic
} 


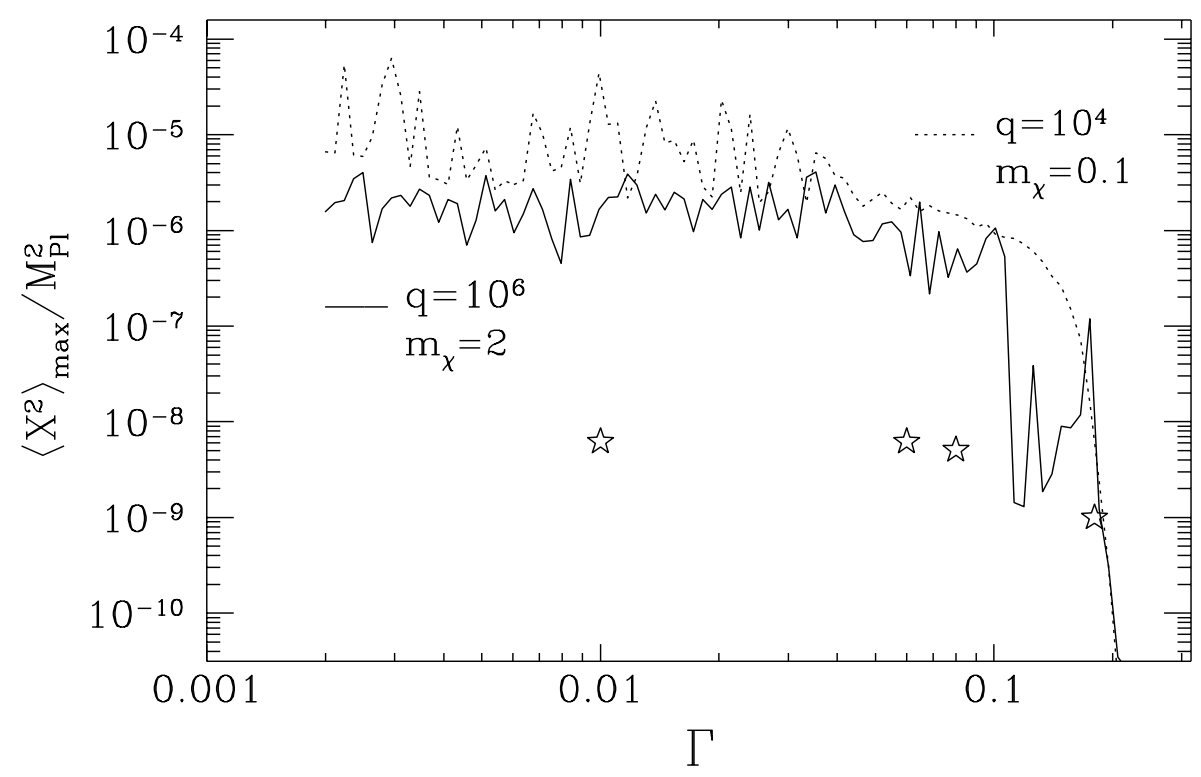

Figure 2: The maximum value of the variance of the $X$-field, $\left\langle X_{\max }^{2}\right\rangle$, is shown as function of $\Gamma$. Stars mark $\left\langle X_{\max }^{2}\right\rangle$ obtained in the full non-linear problem. $\left\langle X_{\max }^{2}\right\rangle$ in the Hartree approximation is shown by the dotted curve for $q=10^{4}, m_{\chi}=0.1$, and by the solid curve for $q=10^{6}, m_{\chi}=2$.

the oscillating inflaton field can no longer compete with $X$-decays due to the non-zero value of $\Gamma$. For comparison, we show in the same figure the case $\Gamma=0$ represented by the dotted curves [14]. In the $\Gamma=0$ case, particle creation is able to compete with the expansion of the universe so that $\left\langle X^{2}\right\rangle$ remains roughly constant. Another novel feature of the case with non-zero $\Gamma$ is the low level of inflaton fluctuations, $\left\langle\delta \phi^{2}\right\rangle(\tau) \ll \phi_{0}^{2}(\tau)$.

Using Eq. (3), we find for the maximum number density of created $X$-particles $n_{X}=\left[4 q \phi_{0}^{2}(10.8) / \phi_{0}^{2}(0)+m_{\chi}^{2}\right]^{1 / 2} m\left\langle X^{2}\right\rangle \approx\left[10^{3}+m_{\chi}^{2}\right]^{1 / 2} m\left\langle X^{2}\right\rangle \approx 30 m\left\langle X^{2}\right\rangle$.

It is easy to understand that if we increase the value of $\Gamma$, the parametric resonance will not be able to compete with the decay of $X$ at earlier times. Moreover, for sufficiently large values of $\Gamma$, the resonance will be shut off in the linear regime. One goal of this paper is to find the boundary of the model parameter space that will result in sufficient $X$-particle creation for successful baryogenesis.

In exploration of parameter space we turn to the Hartree approximation (for details approximation is valid, while non-adiabatic amplification occurs in the region of the peaks of $\left\langle X^{2}(\tau)\right\rangle$ 114. 
see Ref. [13]), which requires much less computing resources. The maximum value of the variance of $X$ reached during the time evolution of the fields in the Hartree approximation is shown in Fig. 2 as a function of the parameters of the model. Here the stars also show the maximum of $\left\langle X^{2}(\tau)\right\rangle$ in the full non-linear problem for a few values of $\Gamma$. At small $\Gamma$ the Hartree approximation overestimates $\left\langle X^{2}\right\rangle$ significantly [13, 14]. Nonetheless, at large values of $\Gamma$ it is a quite reliable approach. We see that $\left\langle X^{2}\right\rangle$ drops sharply when $\Gamma>0.2$, and we have checked that this critical value of $\Gamma$ does not depend significantly upon $m_{X}$ or $q$.

The most relevant case with $q=10^{8}$, where $X$-bosons as massive as ten times the inflaton mass can be created, is shown in Fig. 3 in the Hartree approximation. Note, that two lower curves which correspond to $\Gamma$ equal to 0.08 and 0.12 never reach the limiting value $\left\langle X^{2}\right\rangle_{\max } \sim 10^{-10} M_{\mathrm{Pl}}^{2}$, which is imposed by rescattering [14, and the Hartree approximation ought to be reliable in this cases.

The final baryon asymmetry depends linearly upon the ratio $\delta$ between the energy stored in the $X$ particles at the end of the preheating stage and the energy stored in the inflaton field at the beginning of the preheating era [16]. From our results, we can estimate that this ratio as

$$
\delta \simeq 3 \times 10^{6} \sqrt{\frac{q}{10^{6}}} m_{\chi} \frac{\left\langle X^{2}\right\rangle}{M_{\mathrm{Pl}}^{2}} .
$$

Therefore, for $q=10^{8}$ and $m_{\chi}=10, \delta$ is of the order of $3 \times 10^{8}\left\langle X^{2}\right\rangle / M_{\mathrm{Pl}}^{2}$. Since the final baryon asymmetry scales approximately as $\Gamma^{-1}$ and is given by $B \simeq 5 \times$ $10^{-4} \delta \epsilon\left(\Gamma / 5 \times 10^{-5}\right)^{-1}$ [16], where $\epsilon$ is an overall parameter accounting for $C P$ violation (it will be typically a one-loop factor times some CP-violating phases), we see that the observed baryon asymmetry $B \simeq 4 \times 10^{-11}$ may be explained by the phenomenon of GUT baryogenesis after preheating if

$$
\frac{\left\langle X^{2}\right\rangle}{M_{\mathrm{Pl}}^{2}} \simeq 5 \times 10^{-13}\left(\frac{10^{-2}}{\epsilon}\right)\left(\frac{\Gamma}{5 \times 10^{-5}}\right) .
$$

From Fig. 3 we can read that this only may happen if $\Gamma_{X}$ is smaller than about $10^{-3} m_{X}$. This result may be considered very comfortable since we can conclude that whenever the resonance develops, i.e., when $\Gamma_{X} \lesssim 10^{-1} m=10^{-2} m_{X}$, GUT baryogenesis after preheating is so efficient that the right amount of baryon asymmetry is produced for 


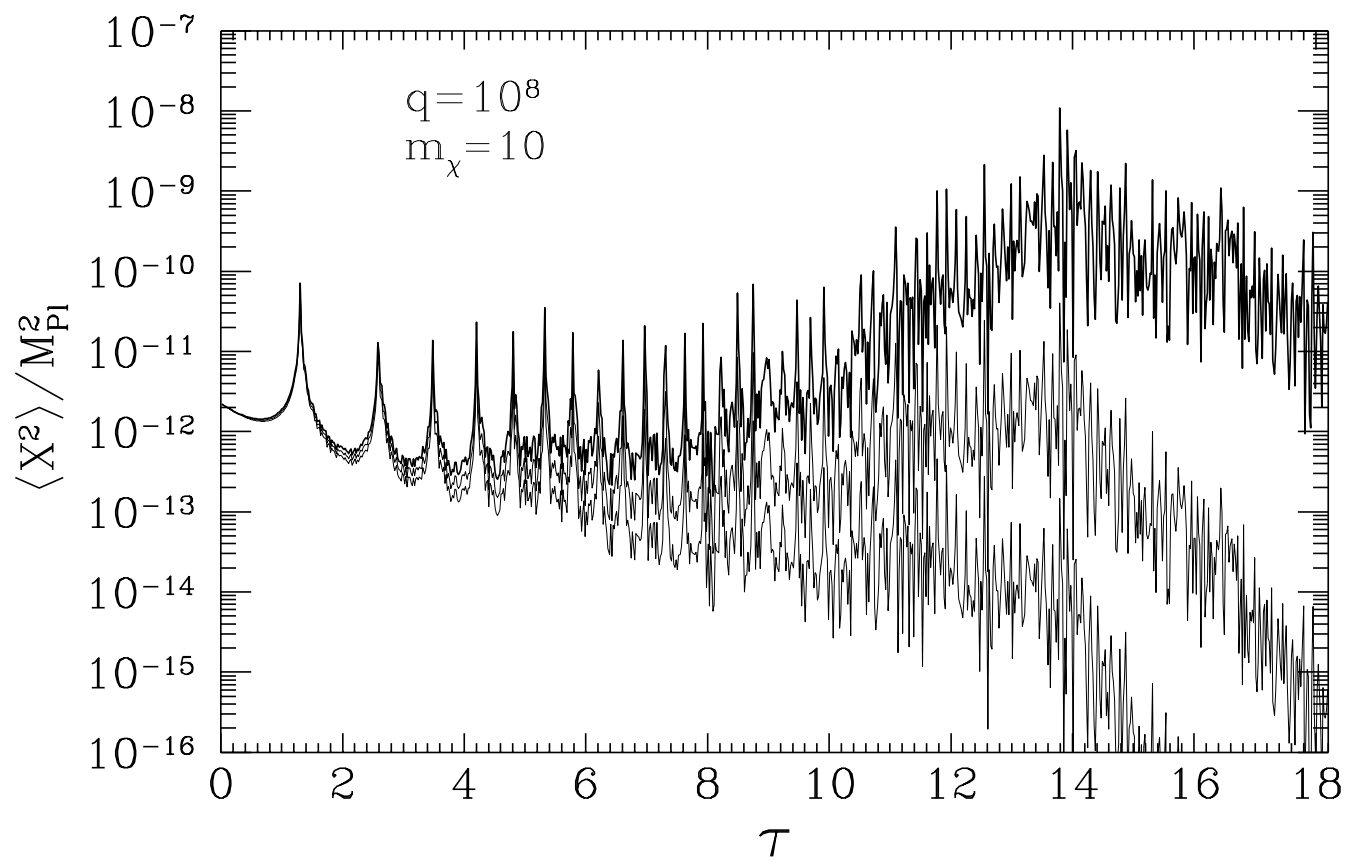

Figure 3: The time dependence of the variance of $X$ in the Hartree approximation with model parameters $q=10^{8}, m_{\chi}=10$ and for three values of $\Gamma$, from top to bottom: $0.04,0.08,0.12$.

almost the entire range of values of the decay rate $\Gamma_{X}$. In other words, provided that superheavy $X$-bosons are produced during the preheating stage, they will be ineffective in producing the baryon asymmetry only if their decay rate falls in the range $10^{-3} m_{X} \lesssim \Gamma_{X} \lesssim 10^{-2} m_{X}$

\section{Conclusions}

From our findings we may infer some phenomenological implications for any model of GUT baryogenesis in preheating. As we already mentioned, $B$ violation through sphaleron transitions are expected to be fast at high temperatures [10], and would erase the baryon asymmetry produced after preheating unless the supermassive $X$ bosons generate some nonvanishing value of $B-L$. A natural way to overcome this problem is to adopt a GUT like $S O(10)$, where an asymmetry in $B-L$ may be generated by the lepton-number violating decays of the Higgs field $\phi_{\mathbf{1 2 6}}$ in the 126-representation of $S O(10)$ which transforms as a singlet under the $S U(5)$ decomposition. 
This Higgs field is responsible for the Majorana masses of the right-handed neutrinos through the symmetric couplings $h_{\nu_{R}} \phi_{\mathbf{1 2 6}} \cdot \nu_{R}^{T} C \nu_{R}$. Requiring that $\Gamma_{\phi_{\mathbf{1 2 6}}}$ is smaller than about $10^{-3} m_{\phi_{126}}$ imposes the constraint $h_{\nu_{R}} \lesssim 10^{-1}$. Through the see-saw mechanism, light neutrinos may therefore get masses in the range $10^{-3}$ to $10^{-2} \mathrm{eV}$ for $h_{\nu_{R}} \sim 10^{-3}$.

Notice that, as opposed to large-angle scattering processes, forward-scatterings do not alter the distribution functions of the particles traversing a gas of quanta, but only alter the dispersion relation. This remains true even in the case of a nonequilibrium system such as the one represented by the gas of $\phi_{\mathbf{1 2 6}}$ particles created in preheating. At the end of reheating the right-handed neutrinos receive a mass of the order of $h_{\nu_{R}}\left\langle\phi_{\mathbf{1 2 6}}^{2}(1)\right\rangle^{1 / 2}$ from non-thermal corrections with the $\phi_{\mathbf{1 2 6}}(1)$-particles due to forwardscatterings. However, this mass is not large enough to suppress kinematically the decay rate of the $\phi_{\mathbf{1 2 6}}$. This would require the energy of the $\phi_{\mathbf{1 2 6}}$ 's to be smaller than $h_{\nu_{R}}\left\langle\phi_{\mathbf{1 2 6}}^{2}(1)\right\rangle^{1 / 2}$, which only happens if $\left(\left\langle\phi_{\mathbf{1 2 6}}^{2}(1)\right\rangle / M_{\mathrm{Pl}}^{2}\right)$ is larger than about $10^{-8} g h_{\nu_{R}}^{-2}$.

In conclusion, we have performed a numerical analysis of the production of superheavy $X$-bosons during the preheating stage following the end of inflation and have shown that the observed baryon asymmetry may be produced in the decay of these non-thermal GUT bosons if the value of their decay rate is smaller than about $10^{-3} m_{X}$. GUT baryogenesis after preheating solves many of the serious drawbacks of GUT baryogenesis in the old theory of reheating where the production of superheavy states after inflation was kinematically impossible. Moreover, the out-of-equilibrium condition is naturally attained in our scenario since the distribution function of the $X$-quanta generated at the resonance is far from a thermal distribution. This situation is considerably different from the one present in the GUT thermal scenario where superheavy particles usually decouple from the thermal bath when still relativistic and then decay producing the baryon asymmetry.

It is quite intriguing that out of all possible ways the parametric resonance may develop, Nature might have chosen only those ways without instantaneous thermalization and also with a successful baryogenesis scenario. 


\section{Acknowledgements}

The work of EWK is supported in part by the Department of Energy and by NASA grant NAG 5-2788. The work of IIT is supported in part by the U.S. Department of Energy under Grant DE-FG02-91ER40681 (Task B) and by the National Science Foundation under Grant PHY-9501458. IIT would like to thank the Theoretical Astrophysics Group at Fermilab and the CERN Theory Division where part of this work was done for their kind hospitality.

\section{References}

[1] A.H. Guth, Phys. Rev. D23, 347 (1981). For a recent review and list of references, see A.D. Linde, Particle Physics and Inflationary Cosmology, (Harwood Academic, New York, 1990).

[2] A. D. Dolgov and A. D. Linde, Phys. Lett. B116, 329 (1982); L. F. Abbott, E. Fahri and M. Wise, Phys. Lett. B117, 29 (1982).

[3] See, for instance, E. W. Kolb and M. S. Turner, The Early Universe, (AddisonWesley, Reading, Ma., 1990).

[4] J. Ellis et al.. Phys. Lett. B118, 335 (1982).

[5] M. Grisaru, W. Siegel and M. Rocek, Nucl. Phys. B159, 429 (1979).

[6] For a review, see E. W. Kolb and M. S. Turner, Ann. Rev. Nucl. Part. Sci. 23, 645 (1983).

[7] E. W. Kolb and S. Wolfram, Nucl. Phys. B172, 224 (1980).

[8] J. Fry, K. A. Olive, and M. S. Turner, Phys. Rev. D22, 2953 (1980).

[9] D. V. Nanopoulos and S. Weinberg, Phys. Rev. D20, 2484 (1979); J. A. Harvey, E. W. Kolb, D. B. Reiss, and S. Wolfram, Nucl. Phys. B201, 16 (1982); T. Yanagida and M. Yoshimura, Nucl. Phys. B168, 534 (1980). 
[10] V. A. Kuzmin, V. A. Rubakov and M. E. Shaposhnikov, Phys. Lett. B155, 36 (1985).

[11] L. A. Kofman, A. D. Linde and A. A. Starobinsky, Phys. Rev. Lett. 73, 3195 (1994).

[12] S.Yu. Khlebnikov and I.I. Tkachev, Phys. Rev. Lett. 77, 219 (1996).

[13] S. Khlebnikov and I. Tkachev, Phys. Lett. B390, 80 (1997).

[14] S. Khlebnikov and I. Tkachev, Phys. Rev. Lett. 79, 1607 (1997).

[15] S. Khlebnikov and I. Tkachev, Phys. Rev. D56, 653 (1997).

[16] E.W. Kolb, A.D. Linde and A. Riotto, Phys. Rev. Lett. 77, 4290 (1996).

[17] B.R. Greene, T. Prokopec and T.G. Roos, Phys. Rev. D56, 6484 (1997).

[18] A. D. Sakharov, JETP Lett. 5, 24 (1967).

[19] T. Prokopec and T.G. Roos, Phys. Rev. D55, 3768 (1997).

[20] S. Kasuya and M. Kawasaki, Phys. Lett. B388, 686 (1996). 